\title{
Modeling Nitrogen Isotope Chemistry in the Solar Nebula
}

\author{
JASMINE GARANI AND JAMES LYONS
}

Arizona State University

Presenting Author: jgarani@asu.edu

Introduction: Analyses of the Genesis samples showed that there is a $400 \%$ difference in the ${ }^{15} \mathrm{~N} /{ }^{14} \mathrm{~N}$ ratio between bulk Earth and the Sun [1]. $\mathrm{N}_{2}$ photodissociation and the process of self-shielding in the solar nebula may account for this large difference in $\mathrm{N}$ isotope ratios. Experiments on $\mathrm{N}_{2} / \mathrm{H}_{2}$ mixtures have demonstrated this possibility, but for an unrealistic $1: 1$ gas ratio [2]. Here, we add $\mathrm{N}$ isotopes and $\mathrm{N}_{2}$ self-shielding to a photochemical solar nebula model.

$\mathbf{N}_{2}$ self-shielding: As UV photons penetrate into a column of nebular gas, the ${ }^{28} \mathrm{~N}_{2}$ become optically thick and dissociates very slowly. However, since ${ }^{29} \mathrm{~N}_{2}$ dissociates at different wavelengths and is much less abundant, ${ }^{29} \mathrm{~N}_{2}$ continues to dissociate resulting in an accumulation of ${ }^{15} \mathrm{~N}$.

Methods: Using a 2-D solar nebula disk model, 717 chemical reactions are modeled through simulated turbulent mixing in a vertical column with1-D diffusion from the mid-plane to the upper UV surface of the disk. 323 new reactions have been added to the original model to account for the new nitrogen chemistry. We explore several key model parameters for the intensity of the radiation field (e), vigor of turbulent mixing $(\alpha)$, and initial molecular cloud values (MCV) of key $\mathrm{N}$ bearing species

Results and Conclusions: We find that a wide range of radiation fields ( $\mathrm{e}=1-100$ times the interstellar field) and turbulence $(\mathrm{a}=.01-.001)$ yield high $\mathrm{d}^{15} \mathrm{~N}$ values in $\mathrm{NH}_{3}$ on dust grains $\left(\mathrm{NH}_{3 g r}\right)$, but that there is a strong dependence on assumed initial cloud values (MCVs) for $\mathrm{HCN}$ and $\mathrm{NH}_{3}$. If $\mathrm{MCV}$ for $\mathrm{NH}_{3}$ was low $\left(<10^{-6}\right)$, then $\mathrm{N}_{2}$ self-shielding in the nebula accounts for the inner solar system enrichment. If $\mathrm{NCV}$ was higher, then $\mathrm{N}_{2}$ self-shielding in the molecular cloud may also have also be needed.

Our new model, with updated nitrogen chemistry, demonstrates that self-shielding of $\mathrm{N}_{2}$ can explain the $400 \%$ enrichment of inner solar system $\mathrm{N}$ isotope ratios. We are currently exploring simultaneous solution for both nitrogen and oxygen isotopes.

References: [1] Furi, E and Marty, B. et al. (2015) Nat Geo, 8, 515. [2] Chakraborty, S. et al. (2014) PNAS, 111, 14704. 